\section{SAT0192 \\ COMPETITIVE ELISA AND BRIDGING ELISA WITH ACID DISSOCIATION DETECT ANTI-DRUG ANTIBODIES IN A GREATER PROPORTION OF PATIENTS TREATED WITH TNF-ALPHA INHIBITORS THAN CLASSICAL BRIDGING ELISA}

M. Ogrič ${ }^{1}$, P. Žigon ${ }^{1}$, K. Lakota ${ }^{1,2}$, S. Praprotnik ${ }^{1}$, D. Drobne ${ }^{3,4}$, B. Štabuc ${ }^{3,4}$, S. Sodin-Semrl' ${ }^{1,2}$, S. Čučnik ${ }^{1,5}$. ${ }^{1}$ Department of Rheumatology, University Medical Centre Ljubljana, Ljubljana, ${ }^{2}$ FAMNIT, University of Primorska, Koper, ${ }^{3}$ Department of Gastroenterology, University Medical Centre Ljubljana, ${ }^{4}$ Faculty of Medicine, ${ }^{5}$ Faculty of Pharmacy, University of Ljubljana, Ljubljana, Slovenia

Background: Therapeutic drug monitoring is used to guide treatment in patients treated with TNF- $\alpha$ inhibitors. Current bridging ELISA (bELISA), mostly used in routine analysis, cannot detect anti-drug antibodies (ADA) in immune complexes and differentiate between neutralizing and non-neutralizing ADA. Reporter Gene Assay (RGA), which detects only neutralizing ADA, is both costly and labourintensive. Therefore, alternative assays are warranted to obviate these limitations.

Objectives: To develop an in-house competitive ELISA (cELISA) for detection of neutralizing $A D A$, to compare results between four different assays for ADA detection and to propose an algorithm to assist clinicians in personalized therapeutic drug monitoring of Infliximab (IFX) and Adalimumab (ADL).

Methods: Samples from 105 patients on IFX or ADL therapy $\left(n_{I F X}=61, n_{A D L}=44\right)$ from the Departments of Rheumatology and Gastroenterology, University Medical Centre Ljubljana, with undetectable drug levels, were tested with in-house cELISA, in-house bELISA, in-house bELISA with acetic acid dissociation (acid bELISA (1)) and RGA. cELISA was developed following the principles of RGA, briefly, diluted samples were pre-incubated with a fixed amount of IFX or ADL, linked to horseradish peroxidase. After incubation on a TNF- $\alpha$ plate, the reaction was detected using TMB substrate. Within and between-run imprecisions for cELISA were determined. Correlation coefficient and agreement between results from different assays were calculated.

Results: Within and between-run imprecisions in CELISA met the validation criteria $(<20 \%)$. We found high correlation between cELISA and RGA (anti-IFX $r=0.932(p<0.0001)$ and anti-ADL $r=0.948(p<0.0001))$ and $100 \%$ agreement between results. CELISA also correlated with bELISA (anti-IFX $r=0.663$ $(p=0.0002)$ and anti-ADL $r=0.896(p<0.0001))$. Agreement between bELISA and CELISA was $79 \%$ for anti-IFX and $82 \%$ for anti-ADL samples.

The more sensitive cELISA and functional RGA detect 13\% (8/61) more positive samples in anti-IFX group and 18\% (8/44) more samples in anti-ADL group. Acid bELISA found $3 \%(2 / 61)$ more positive samples in anti-IFX group and $14 \%(6 / 44)$ of samples in the anti-ADL group.

In total, 16\% (10/61) more samples in anti-IFX and 30\% (13/44) more samples in anti-ADL group were confirmed having ADA. Based on our results we propose a sequential algorithm, which enables reliable, affordable and increased detection of ADA (figure 1)

\section{Therapeutic drug monitoring of Infliximab and Adalimumab}

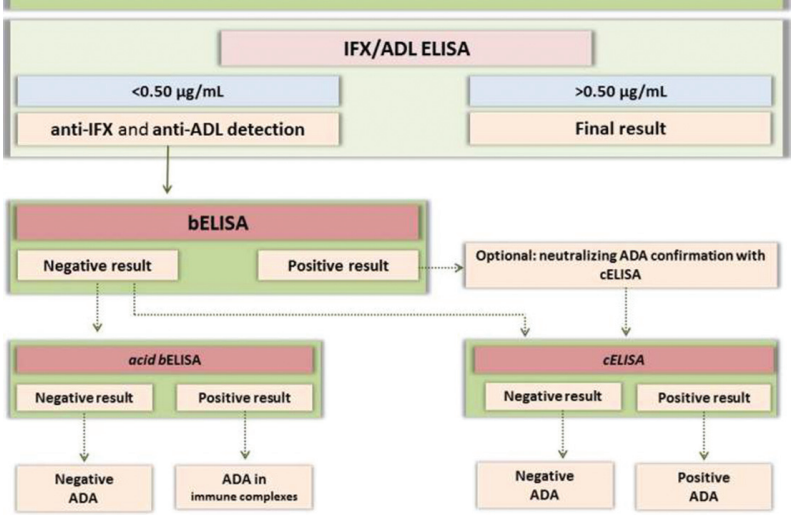

Figure 1. Algorithm for therapeutic drug monitoring of IFX and ADL. IFX - Infliximab, ADL Adalimumab, ADA - anti-drug antibodies, bELISA - bridging ELISA, acid bELISA - bELISA with acid dissociation, cELISA - competitive ELISA;

Conclusions: cELISA and acid bELISA, together, can detect ADA in 16\% more samples in anti-IFX and $30 \%$ in anti-ADL group than classical bELISA used in current practice. The proposed algorithm can assist in everyday practice and enables better evaluation of patients treated with TNF- $\alpha$ inhibitors.
REFERENCE:

[1] Van Stappen, et al. Drug Test Anal 2017;9(2):243-7.

Disclosure of Interest: None declared

DOI: 10.1136/annrheumdis-2018-eular.6486

\section{SAT0193 EARLY DISCONTINUATION OF FIRST LINE BIOLOGICAL TREATMENT WITH ETANERCEPT IN PATIENTS WITH RHEUMATOID ARTHRITIS: RESULTS FROM THE ITALIAN GISEA REGISTRY}

M. Sebastiani ${ }^{1}$, A. Manfredi ${ }^{1}$ on behalf of GISEA, F. lannone ${ }^{2}$, E. Gremese ${ }^{3}$,

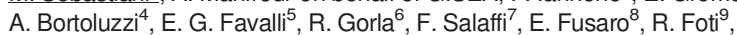

L. Cantarini ${ }^{10}$, R. Caporali ${ }^{11}$, A. Cauli ${ }^{12}$, S. Alivernini ${ }^{3}$, F. P. Cantatore ${ }^{13}$,

A. Carletto ${ }^{14}$, F. Conti ${ }^{15}$, S. D'Angelo ${ }^{16}$, O. Epis ${ }^{17}$, G. Cassone ${ }^{1}$, R. Ramonda ${ }^{18}$

V. Grosso ${ }^{11}$, P. Sarzi-Puttini ${ }^{19}$, G. Ferraccioli ${ }^{3}$, G. Lapadula ${ }^{2}$ on behalf of GISEA'

(Gruppo Italiano Studio Early Arthritis). ${ }^{1}$ Rheumatology Unit, University of Modena and Reggio Emilia, Modena, ${ }^{2}$ Rheumatology Unit, University of Bari, Bari,

${ }^{3}$ Rheumatology Unit, Catholic University of the Sacred Heart, Rome,

${ }^{4}$ Rheumatology Unit, University of Ferrara, Ferrara, ${ }^{5}$ Department of Rheumatology, Gaetano Pini Institute, Milano, ${ }^{6}$ Rheumatology Unit, Spedali civili di Brescia, Brescia, ${ }^{7}$ Rheumatology Unit, Università Politecnica delle Marche, Jesi,

${ }^{8}$ Rheumatology Unit, AOU Città della Salute e della Scienza, Torino,

${ }^{9}$ Rheumatology Unit, Policlinico Vittorio Emanuele, Catania, ${ }^{10}$ Rheumatology Unit, University of Siena, Siena, ${ }^{11}$ Rheumatology Unit, University of Pavia, Pavia,

${ }^{12}$ Rheumatology Unit, University of Cagliari, Cagliari, ${ }^{13}$ Rheumatology Unit, Azienda Ospedaliero-Universitaria Ospedali Riuniti, Foggia, ${ }^{14}$ Rheumatology Unit, University of Verona, Verona, ${ }^{15}$ Rheumatology Unit, Sapienza University of Rome, Rome, ${ }^{16}$ Rheumatology Unit, Rheumatology Institute of Lucania, Potenza,

${ }^{17}$ Rheumatology Unit, Niguarda Hospital, Milano, ${ }^{18}$ Rheumatology Unit, University of Padova, Padova, ${ }^{19}$ Rheumatology Unit, Sacco Hospital, Milano, Italy

Background: Tumor necrosis factor- $\alpha$ inhibitors (TNFi) are usually the first biologic drugs employed in rheumatoid arthritis (RA) after failure of conventional disease-modifying antirheumatic drugs (DMARDs). Retention rate is a useful surrogate marker of effectiveness and safety in real life, but few studies investigated the causes of early discontinuation of these drugs.

Objectives: Aim of the study was to investigate the possible predictors of early discontinuation (within 1 year of treatment) of etanercept (ETA) in RA patients enrolled into the GISEA (Italian Group for the Study of Early Arthritis) registry.

Methods: RA patients who began etanercept as first biologic DMARD were included in the study. For all patients age, sex, disease duration, smoking status, the intake of glucocorticoids and DMARD, clinical and serological data, comorbidities and extra-articular manifestations were collected.

Results: We analyzed 477 RA patients (females/males 382/95, mean age 51.3 \pm 14.1 years; mean DAS28 5.4 \pm 1.5 ); rheumatoid factor (RF) and anti-citrullinated peptide antibodies (ACPA) were positive in $66 \%$ and $62.3 \%$, respectively. Comorbidities were observed in $16.6 \%$ of patients, mainly cardiovascular diseases, while extra-articular RA manifestations were recorded in 6.3\%. Concurrent DMARDs therapies were reported in $54.3 \%$ of patients, mainly methotrexate $(40.5 \%)$, while $52.4 \%$ of subjects taken low doses of steroids.

Seventy patients (14.7\%) discontinued ETA during the first year (for inefficacy in 43 patients, adverse events in 22, and other reasons in 5). The presence of comorbidities and a combination therapy with DMARDs different by MTX were independent predictors of early discontinuation of ETA at multivariate analysis (see table 1). The association with MTX didn't increase the 1-year retention rate of ETA. No significant associations were observed with steroids, presence of RF or ACPA or the disease activity at baseline.

Table 1. Multivariate analysis. Factors associated to early discontinuation of etanercept

\begin{tabular}{lllll}
\hline Parameter & $\begin{array}{l}\text { Standard } \\
\text { Error }\end{array}$ & $\begin{array}{l}\text { Odds } \\
\text { ratio }\end{array}$ & $\begin{array}{l}\text { 95\% Confidence } \\
\text { Interval }\end{array}$ & $p$ \\
\hline Comorbidities & 0,28 & 1,86 & $1.07-3.25$ & 0,029 \\
$\begin{array}{l}\text { DMARDs different by } \\
\text { MTX }\end{array}$ & 0,35 & 2,01 & $1.014-3.982$ & 0,045 \\
ETA monotherapy & 0,297 & 0,701 & $0.392-1.256$ & 0,232 \\
\hline
\end{tabular}

Conclusions: ETA demonstrated a high persistence in RA patients and after 12 months more than $85 \%$ of patients continued the treatment. The presence of comorbidities and a combination therapy with DMARDs different by MTX were associated to an early withdrawal of the drug.

Disclosure of Interest: None declared

DOI: 10.1136/annrheumdis-2018-eular.3642 